Acta Cryst. (2002). A58 (Supplement), C226

FROM VERY WEAK TO VERY STRONG HYDROGEN BONDS TESTING THE LIMITS AND FINDING APPLICATIONS USING HALOGENS

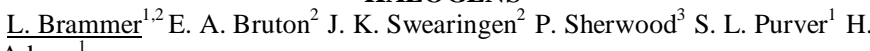
Adams ${ }^{1}$

${ }^{1}$ Department of Chemistry, University of Sheffield, Sheffield S3 7HF, UK ${ }^{2}$ Department of Chemistry \& Biochemistry, University of Missouri-St. Louis, 8001 Natural Bridge Road, St. Louis, MO 63121-4499, USA ${ }^{3}$ Department of Computational Science and Engineering, CLRC Daresbury Laboratory, Daresbury, Warrington WA4 4AD, UK

Hydrogen bonds involving halogens cover a remarkable range of strengths from the very strongest at ca. $40 \mathrm{kcal} / \mathrm{mol}$ in the bifluoride ion $[\mathrm{F}-\mathrm{H}-\mathrm{F}]$ down to $<1 \mathrm{kcal} / \mathrm{mol}$ calculated for $\mathrm{C}-\mathrm{H}^{\cdots} \mathrm{F}$ hydrogen bonds. While the upper limit is fairly well defined the lower limit of defining hydrogen bonds has long been open to debate. Our studies of $\mathrm{D}-\mathrm{H}^{\cdots} \mathrm{X}-\mathrm{C}(\mathrm{D}=\mathrm{C}, \mathrm{N}, \mathrm{O} ; \mathrm{X}=\mathrm{F}, \mathrm{Cl}, \mathrm{Br}, \mathrm{I})$ interactions using database analysis of geometries combined with ab initio computational interpretation suggest weak hydrogen bond behavior. There are useful comparisons to be made with stronger hydrogen bonds formed by metal halides, i.e. $\mathrm{D}-\mathrm{H}^{\cdots} \mathrm{X}-\mathrm{M}(\mathrm{M}=$ transition or main group metal), which show similar but more well-defined geometric behavior. Furthermore, cis and fac coordinated halide ligands $\left(\mathrm{MX}_{2}\right.$ and $\mathrm{MX}_{3}$, respectively) exhibit a cooperative effect to yield binding pockets for hydrogen bond donors (D-H). The exploitation of such directional interactions using perhalometallate ions combined with cationic hydrogen bond donors allows the design of ionic hydrogen-bonded network solids. Illustrative examples in which the anions serve as network nodes will be presented and some challenges facing this application to inorganic crystal engineering will be outlined.

Keywords: HYDROGEN BONDS HALOGENS CRYSTAL ENGINEERING

Acta Cryst. (2002). A58 (Supplement), C226

CAN HYDROGEN BE OFF-CENTRED EVEN IN A CENTROSYMMETRIC ENVIRONMENT?

I. Olovsson ${ }^{1}$ H. Ptasiewicz-Bak ${ }^{2}$ T. Gustafsson ${ }^{1}$ I. Majerz ${ }^{3}$

${ }^{1}$ University of Uppsala, Uppsala, Sweden ${ }^{2}$ Institute of Nuclear Chemistry and

Technology, Warszawa, Poland ${ }^{3}$ Wroclaw University, Wroclaw, Poland

The hydrogen distribution in KHdichloromaleate has been determined by neutron diffraction at 30,90, 170 and $295 \mathrm{~K}$. There are two independent hydrogens in two short H-bonds (around $2.45 \AA$ ). All atoms may be refined successfully in $P-1$ at all temperatures, with hydrogen located at the symmetry centres. However, an unconventional and less restricted refinement, with the non-hydrogen atoms kept in $P-1$ but with the protons refined without this restriction in $P 1$, gives a very interesting result: The non-hydrogen atom structure is still centrosymmetric, whereas the hydrogens are off-centred! Statistical significance tests also clearly show that a non-centrosymmetric hydrogen distribution gives the correct description of the structure. This is a very important conclusion which does not at all agree with traditional ideas about the situation in short hydrogen bonds: it is normally assumed that the hydrogen distribution adopts the same symmetry as the 'heavy atoms'. Another very surprising result is that the hydrogen distribution is different at $30 \mathrm{~K}$ and $90 \mathrm{~K}$ as compared to at $170 \mathrm{~K}$ and $295 \mathrm{~K}$, although the hydrogen bonds are equally long: at 30 and $90 \mathrm{~K}$ both hydrogens are closer to one and the same molecule (M) whereas at 170 and $295 \mathrm{~K}$ one hydrogen is closer to $\mathrm{M}$ and the other closer to $\mathrm{Mc}$ ( $\mathrm{Mc}$ is related to $\mathrm{M}$ by the centre of symmetry). There is evidently a subtle structural change between 90 and $170 \mathrm{~K}$, which is also indicated by the cell dimensions: The derivatives of the cell parameters as a function of temperature change abruptly around $135 \mathrm{~K}$ !

Keywords: KHDICHLOROMALEATE, NEUTRON DIFFRACTION, SHORT HYDROGEN BONDS
Acta Cryst. (2002). A58 (Supplement), C226

\section{ENFORCED FOLDING BY LOCALIZED INTRAMOLECULAR HYDROGEN BONDS: HOLLOW HELICES WITH TUNABLE CAVITY SIZE}

B. Gong

State University of New York at Buffalo Department of Chemistry 811

Natural Sciences Complex BUFFALO NEW YORK 14260 USA

Folding oligomers with backbones that are rigidified by localized three-center hydrogen bonding interactions have been developed. The three-center, intramolecular hydrogen bonds are stable in polar as well as non-polar solvents. These oligomers adopt folded, highly stable conformations in both solid state and solution. Unlike most known foldamers, the folding of these oligomers is an inherent property of their backbones, and is thus independent of their size. Changing side chains does not affect the folded conformation. An oligomer adopts either a crescent or helical conformation depending on its length. The helices contain large, tunable interior cavities. Longer oligomers and polymers based on the same design principle fold into helical nanotubes with tunable cavity size and modifiable outside surfaces.

\section{Keywords: HYDROGEN BOND FOLDING NANOCAVITY}

Acta Cryst. (2002). A58 (Supplement), C226

\section{STEREOSPECIFIC INTERACTIONS OF HISTIDINE WITH OTHER PLANAR GROUPS IN PROTEIN STRUCTURES}

P. Chakrabarti R. Bhattacharyya R. Saha U. Samanta

Bose Institute Biochemistry P-1/12 CIT Scheme VIIM CALCUTTA WEST BENGAL 700054 INDIA

Aromatic-aromatic interactions are known to contribute to the stability of protein structures. Earlier studies have shown a preference for the edge-to-face interaction geometry. Among the aromatic residues histidine (His) is unique, as it can exist in the neutral or positively charged form at the neutral $\mathrm{pH}$. As such it can interact with other aromatic residues as well as form hydrogen bonds with polar and charged (both negative and positive) residues. We have analyzed the geometry of interaction (indicated by the closest contact being less than $4 \AA$ ) of His residues with nine other planar side chains containing aromatic (residues Phe, Tyr, Trp and His), carboxylate (Asp and Glu), carboxamide (Asn and Gln) and guanidinium (Arg) groups in 432 polypeptide chains. Except for the aspartic (Asp) and glutamic(Glu) acid side-chains, all other residues prefer to interact in a face-to face or offset-face-stacked orientation with the His ring. Carboxylate groups, forming hydrogen bonds with His are found mostly in the perpendicular geometry. The geometry of aromatic-aromatic interaction is found to depend on the relative location in a particular secondary structure. For example, in a helix it depends on the sequence difference between the interacting pair. Specific interactions $\left(\mathrm{C}-\mathrm{H}^{\cdots}{ }^{*} \pi\right.$ and $\mathrm{N}-\mathrm{H} \cdot{ }^{\prime \prime} \pi$ ) that result from this geometry may cause a given pair of residues (such as Phe-His) with a particular sequence difference to occur more than expected.

Keywords: INTERACTION-GEOMETRY, HYDROGEN-BONDING, AROMATIC-AROMATIC-INTERACTION 